\title{
Myelofibrosis in a Child with Tuberculosis: A Case Report
}

Malla $\mathbf{K}^{1}$, Malla $\mathbf{T}^{2}$, Thaplial $\mathbf{A}^{3}$

${ }^{1}$ Dr. Kalpana Malla MD Pediatrics, Assistant Professor, ${ }^{2}$ DrTejesh Malla MD Paediatrics, Assistant Professor, ${ }^{3}$ Dr. Anna Thapalial, MD Paediatrics, Professor and HOD,Pediatrics, Manipal College of Medical Sciences,(MCOMS), Pokhara, Nepal.

Address for Correspondence: Dr. Kalpana K Malla M.D., A-7,Staff Quarters, Manipal Teaching hospital, Phulbari, Pokhara, Nepal. Ph:00977-61-526416 Ext:167(Res), 119 (Off.)

E-mail: kalpana17@hotmail.com

\begin{abstract}
Myelofibrosis (MF), or fibrosis of the bone marrow, is an uncommon condition in children. Fewer than 100 cases have been described in the medical literature. Most cases in children arise secondary to other disease processes. We present a case of Myelofibrosis in a 12-year-old girl. The purpose of reporting this case is that this child had a diagnostic dilemma and was sent home with poor prognosis but with proper diagnosis and treatment she improved and may have a complete cure, as the myelofibrosis was secondary to tuberculosis.
\end{abstract}

Key words: myelofibrosis, tuberculosis, acute megakaryoblastic leukemia (AMKL)

\section{Case Report}

A 9 Year old female child from Tansen, a western region of Nepal was admitted to Manipal Teaching Hospital with complaints of abdominal distension for $1 \frac{1}{2}$ months, generalised anasarca for two weeks and non passage of stool for two days along with frequent bile stained vomiting. On examination she had features of intestinal obstruction. She also had peticheal and purpuric rashes all over her body. On further inquiry there was a past history of insidious onset of low-grade intermittent fever for two months, and similar rashes for one-month duration. For these problems she was taken to a hospital where malignancy was suspected and was referred to cancer hospital where bone marrow aspiration was attempted with no results. Bone Marrow Biopsy was not done.The diagnosis was assumed to be aplastic anemia. She was given one unit blood transfusion and discharged home after explaining about the poor prognosis of disease. Her condition remained same at home and was brought here after she developed intestinal obstruction. She was not immunized and was the $9^{\text {th }}$ child of a poor farmer. Her grandfather was a diagnosed case of sputum positive tuberculosis and died one year ago.

On examination She was ill looking, pale and cachexic (Fig.1). Her vitals were stable, was febrile with temperature of $100^{\circ} \mathrm{F}$. She had firm cervical lymphadenopathy with a few matted nodes, she had bilateral pitting ankle oedema

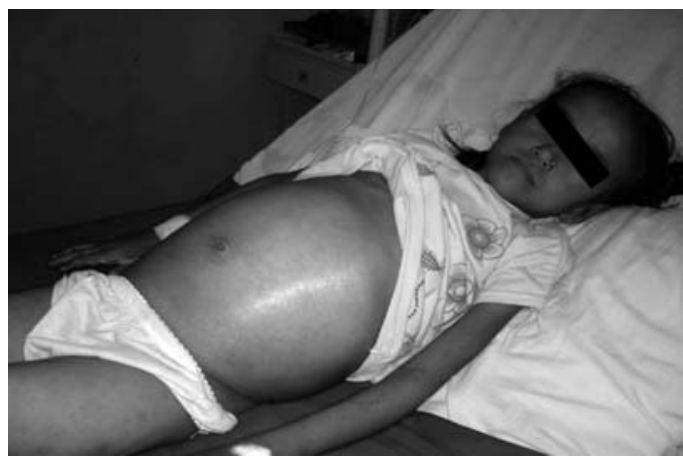

Fig 1: Showing a cachexic child with abdominal distension and skin survey revealed petechiae, purpura all over the body. Anthropometry revealed a wasted figure with weight $20 \mathrm{kgs}$ in spite of oedema, MAC- $12 \mathrm{~cm}$. Abdomen was hugely distended with shiny skin and dilated visible veins. Abdomen was tender to Palpation. She had Hepatomegaly $3 \mathrm{~cm}$, and Speelnomegaly $-5 \mathrm{~cm}$, Abdominal girth $69 \mathrm{~cm}$, Fluid thrill was present, confirming the presence of ascites. Bowel sound was sluggish on auscultation. Examination of the respiratory system showed normal vesicular breath sounds with crepitations on both lung fields. Other systems were normal. Laboratory investigations showed pancytopenia with total count 1800/cumm, haemoglobin 4.9 gm $\%$ and platelets-14,000/cumm.Peripheral smear showed microcytic,hypo chromic anaemia and there were no blast cells. ESR was $13 \mathrm{~mm}$ in 1st hr. Plain X-ray abdomen erect revealed sub acute obstruction (fig 2). Ascitic fluid tap was straw coloured, Cell count 2000/cumm ( $N=30 \%$, L $=70 \%$ ), Smear showed -pus cell, Gram positive cocci in clusters, Protein- $2.7 \mathrm{gm} / \mathrm{dl}$, Sugar $-45 \mathrm{mg} / \mathrm{dl}$, staph aureus was isolated in Ascitic fluid Culture. She also had Urinary Tract Infection with significant growth of (>105 CFU/ml) of Klebsiella and E.coli, Total serum protein was $5.9 \mathrm{gm} \%$, Serum Albumin - $1.9 \mathrm{mg} / \mathrm{dl}$, HIV spot test was negative gastric levage for AFB was negative, Matoux test negative, BCG Acceleration test was positive with an induration of more than $15 \mathrm{~mm}$, USG Abdomen showed hepatomegaly with fatty

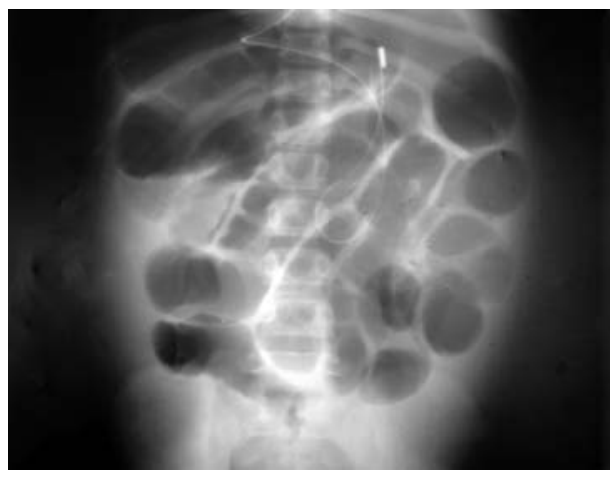

Fig 2: Plain X-ray abdomen erect view showing signs of sub acute obstruction. 
changes and massive ascites, Spleen was not seen due to a poor window. Bone marrow study aspiration was a dry tap and biopsy revealed Myelofibrosis. Based on low-grade fever, contact with tuberculosis, un immunized child, positive BCG acceleration test and bone marrow biopsy findings final diagnosis of Myelofibrosis due to Disseminated tuberculosis with sub acute intestinal obstruction and peritonitis was made.

Due to poor general condition and pancytopenia conservative management was planned for sub acute obstruction. She improved with treatment. Blood was transfused three times and anti TB treatment was started under category I regime with $2 \mathrm{HRZE} / 6 \mathrm{HR}$ along with Prednisolone. With this treatment she showed a phenomenal improvement. Her general well being, improved within seven days. Her abdominal girth decreased from $69 \mathrm{~cm}$ to $57 \mathrm{~cm}$, her appetite increased and oedema and fever also subsided. She has completed her 3 months treatment. Hepatospleenomegaly (liver $-1 \mathrm{~cm} \&$ spleen just palpable) has regressed and Blood counts were normal with total count of $9800 /$ cumm, haemoglobin $12 \mathrm{gm} \%$ and platelets-2 40,000/ cumm and normal Peripheral smear.

\section{Discussion}

Myelofibrosis (MF), alternatively is also known as Myelosclerosis, Acute Myelofibrosis Of Childhood, Idiopathic Myelofibrosis, Agnogenic Myeloid Metaplasia (AMM), Aleukemic Megakaryocytic Myelosis (ALMM) ${ }^{1}$. Fewer than 100 cases of paediatric MF have been reported worldwide $^{2}$. Internationally Cases of paediatric MF have been described in association with tuberculosis (in sudan) ${ }^{3}$, and visceral leishmaniasis (in Pakistan) ${ }^{4}$. Females outnumber males by a ratio of approximately $2: 1$. Children younger than 3 years have been reported ${ }^{2}$.

Myelofibrosis is frequently associated with malignancy -Acute Megakaryoblastic Leukaemia [AMKL]). Other associations are Prior to a clear diagnosis of Acute Leukaemia, Multiple Myeloma, Myeloproliferative Diseases, Hodgkin's Disease and Non-Hodgkin's Lymphoma ${ }^{5}$. Numerous nonmalignant diseases have also been reported. They are infections like Tuberculosis and Visceral Leishmaniasis,3,4. Renal Osteodystrophy, Rickets,Down Syndrome have been reported too ${ }^{6,7}$. In children Myelofibrosisis is classified as, Primary (idiopathic), Secondary (malignant), Secondary (non-malignant) $^{8}$ The common Symptoms and signs are Fatigue, Weight Loss, Fever, Sweating, Weakness, Palpitations of the Heart, Shortness of Breath,Abdominal pain or discomfort,Unexpected bleeding,Bone pain, especially in the legs, Pallor (anaemia),Oral thrush (neutropenia), Spleenomegaly (frequent) or hepatomegaly or lymphadenopathy (less common) ${ }^{8}$.

Haematology reveals Anaemia or thrombocytopenia or both, with or without leukocytosis. On peripheral blood smear Red blood cells are normochromic-normocytic with mild poikilocytosis. Reticulocytosis, nucleated RBCs, tear drops and fragments maybe present ${ }^{9}$. Bone marrow aspirate is usually a dry tap. Bone marrow biopsy typically exhibits marked fibrosis with pockets of cellularity that have fibroblasts and atypical megakaryoblasts. The hallmark is increased reticulin staining in bone marrow biopsy. The fibrous network observed is collagenous and contains fibronectin ${ }^{10}$. Neutrophil Alkaline Phosphatase (LAP) score may give a hint to the diagnosis. A low level suggests Chronic Myeloid Leukaemia and a high level suggests chronic inflammation ${ }^{11}$.

No definite therapy has been evaluated. Bone marrow transplantation is curative in some cases ${ }^{12}$. Many asymptomatic patients, if stable, do not require treatment. Therapy is directed to manage complications and to improve quality of life. The identification of cause like Rickets and Tuberculosis should prompt treatment of that disorder. For younger patients allogeneic marrow transplantation should be considered $^{12}$. Other therapeutic options are Corticosteroids, IV Immunoglobulins, Alfa Interferon, which may lead to resolution of fibrosis and restoration of blood counts ${ }^{13}$. Use of Hydroxyurea, Thalidomide, alone, or in combination with Prednisolone are other options ${ }^{14}$. This therapy showed improvements in anaemia [42\%],thrombocytopenia [77\%] and spleenomegaly $[17 \%]^{15}$ Treatment with Vitamin D, Highdose Methylprednisolone or Interferon Alfa may result in a temporary amelioration of the disease ${ }^{16}$.

The prognosis of childhood MF is variable, depending upon the clinical context in which it occurs. It progresses very rapidly in children. With appropriate treatment for rickets, tuberculosis, systemic lupus erythematosus, Secondary MF may resolve completely spontaneously ${ }^{17}$.

To conclude childhood Myelofibrosis is usually associated with secondary cause, which can resolve completely with appropriate treatment.

\section{References}

1. Boxer LA, Camitta BM, Berenberg W: MyelofibrosisMyeloid Metaplasia In Childhood. Pediatrics 1975 Jun; 55(6): 861-5.

2. Brovall C, Mitchell H, Saral R: Acute Myelofibrosis In A Child. J Pediatr 1983 Jul; 103(1): 91-3.

3. Hashim MS, Kordofani AY, el Dabi MA: Tuberculosis And Myelofibrosis In Children: a report. Ann Trop Paediatr 1997 Mar; 17(1): 61-5.

4. Saleem M, Anwar M, Khan AH: Myelofibrosis In Visceral Leishmaniasis. Br J Haematol 1991 Aug; 78(4): 573-9.

5. Hung IJ, Kuo TT, Sun CF: Subcutaneous Panniculitic TCell Lymphoma Developing In A Child With Idiopathic Myelofibrosis. J Pediatr Hematol Oncol 1999 Jan-Feb; 21(1): 38-41.

6. Ozsoylu S. Rickets And Myelofibrosis. Eur J Pediatr. $2000 \mathrm{Jul} ; 159(7): 544$.

7. Pantazis CG, McKie VC, Sabio H, Davis PC, Allsbrook WC Down's Syndrome And Acute Myelofibrosis. Time Study Of DNA Content During The Progression To Leukemia. Cancer.1988 Jun 1;61(11):2239-43. 
8. Manoharan A: Idiopathic Myelofibrosis: A Clinical Review. Int J Hematol 1998 Dec; 68(4): 355-629.

9. Cervantes F, Alvarez-Larran A, Talarn C, Gomez M, Montserrat E. Myelofibrosis With Myeloid Metaplasia Following Essential Thrombocythaemia: Actuarial Probability, Presenting Characteristics And Evolution In A Series Of 195 Patients Br J Haematol. 2002 Sep;118(3):786-90.

10. R Burkhardt, R Bartl, K Jäger, B Frisch, G Kettner, G Mahl, and M Sund :J Clin Pathol. 1986 March; 39(3): 237-252.

11. Reilly JT: Idiopathic Myelofibrosis: Pathogenesis, Natural History And Management. Blood Rev 1997 Dec; 11(4): 233-42

12. Deeg HJ, Gooley TA, Flowers ME, Sale GE, Allogeneic Hematopoietic Stem Cell Transplantation For Myelofibrosis. Blood. 2003 Dec 1;102(12):3912-8. .
13. Pilorget $\mathrm{H}$, Bangui A, Adam $\mathrm{M}$ : Myelofibrosis Regressing Under Corticotherapy And Intravenous Immunoglobulins In An Infant. Arch Pediatr 1996 Jan; 3(1): 40-3.

14. Mesa RA, Elliott MA, Schroeder G: Durable Responses To Thalidomide-Based Drug Therapy For Myelofibrosis With Myeloid Metaplasia. Mayo Clin Proc 2004 Jul; 79(7): 883-9.

15. McCarthy DM: Annotation. Fibrosis of The Bone Marrow: Content and Causes. Br J Haematol 1985 Jan; 59(1): $1-7$.

16. Ozsoylu S, Ruacan S: High-Dose Intravenous Corticosteroid Treatment In Childhood Idiopathic Myelofibrosis. Acta Haematol 1986; 75(1): 49-51.

17. Sah A, Minford A,Parapia LA. Spontaneous Remission Of Juvenile Idiopathic Myelofibrosis.Br Haematol. 2001 Mar;112(4):1083. 